\title{
An NMR study on the intrinsically disordered core transactivation domain of human glucocorticoid receptor
}

\author{
Do-Hyoung Kim ${ }^{1}$, Anthony Wright ${ }^{2}$ \& Kyou-Hoon Han $^{1,3, *}$ \\ ${ }^{1}$ Genome Editing Research Center, Korea Research Institute of Bioscience and Biotechnology, Daejeon 34141, Korea, ${ }^{2}$ Clinical Research \\ Center, Department of Laboratory Medicine, Karolinska Institutet, Stockholm, Sweden, ${ }^{3}$ Department of Nano and Bioinformatics, \\ University of Science and Technology, Daejeon 34113, Korea
}

\begin{abstract}
A large number of transcriptional activation domains (TADs) are intrinsically unstructured, meaning they are devoid of a three-dimensional structure. The fact that these TADs are transcriptionally active without forming a 3-D structure raises the question of what features in these domains enable them to function. One of two TADs in human glucocorticoid receptor (hGR) is located at its $\mathrm{N}$-terminus and is responsible for $\sim 70 \%$ of the transcriptional activity of hGR. This 58-residue intrinsically-disordered TAD, named tau1c in an earlier study, was shown to form three helices under trifluoroethanol, which might be important for its activity. We carried out heteronuclear multi-dimensional NMR experiments on hGR tau1c in a more physiological aqueous buffer solution and found that it forms three helices that are $\sim \mathbf{3 0} \%$ pre-populated. Since pre-populated helices in several TADs were shown to be key elements for transcriptional activity, the three pre-formed helices in hGR tau1c delineated in this study should be critical determinants of the transcriptional activity of hGR. The presence of prestructured helices in hGR tau1c strongly suggests that the existence of pre-structured motifs in target-unbound TADs is a very broad phenomenon. [BMB Reports 2017; 50(10): 522-527]
\end{abstract}

\section{INTRODUCTION}

Although many globular proteins are three-dimensionally structured, they contain short flexible/disordered loops composed of less than 20 amino acid residues (1). This phenomenon of so-called protein disorder has been known for decades, but since the late 1990s, we have begun encountering

${ }^{*}$ Corresponding author. Tel: +82-42-860-4250; Fax: +82-42-8604259; E-mail: khhan600@kribb.re.kr

https://doi.org/10.5483/BMBRep.2017.50.10.152

Received 7 August 2017, Revised 24 August 2017, Accepted 9 September 2017

Keywords: Human glucocorticoid receptor (hGR), Intrinsically disordered protein (IDP), Nuclear magnetic resonance (NMR), Prestructured motif (PreSMo) some peculiar proteins that contain long unfolded/disordered regions (more than 40 and up to hundreds of residues) that do not form 3-D structures $(2,3)$. These proteins are now named as intrinsically disordered proteins (IDPs) $(4,5)$ and represent a special case of protein disorder. IDPs are highly unorthodox because even without three-dimensional structures, they are capable of performing specific biological functions (including transcription, translation, chaperoning, and cell cycle regulation) or are responsible for many fatal diseases (such as cancers, prion diseases, neurodegenerative diseases including Alzheimer's and Parkinson's, and so on) (6-9). Many viral proteins in HIV-1, HBV, HCV, SARS virus, and Al virus are also IDPs or contain intrinsically disordered regions (IDRs) (4, 10-13).

The unexpected correlation between the "unstructured" nature of IDPs/IDRs and their functionality ended up nullifying the decades-old structure-function paradigm, 3-D structure = function, in protein science and structural biology. Approximately $40 \%$ of the entire protein kingdom is predicted to consist of IDPs/IDRs (11), and the proportion of IDPs/IDRs is much higher $(\sim 60 \%)$ in transcription factors $(14)$. In the case of globular proteins, the collective structural features (secondary, tertiary and quaternary) provide a reasonable explanation of function. However, the absence of tertiary structures in IDPs makes it quite challenging to come up with an explanation on why and how they should function at all. For example, we still do not have a clear understanding on how IDPs bind to their targets.

Initially, IDPs/IDRs were erroneously thought to be completely unstructured (CU) without any trace of secondary structures $(15,16)$. In contrast to this early view, a more quantitative structural picture on IDPs/IDR has emerged from many high-resolution multi-dimensional NMR investigations conducted over the last two decades. These studies have revealed that at least $\sim 70 \%$ of IDPs/IDRs are not fully "unstructured", but contain transient local structural elements in their free state that mediate binding of IDPs to targets (4). The IDPs/IDRs containing transient local structural elements are therefore described to be in a mostly unstructured (MU) state (3) since they are not completely unstructured in terms of secondary structure. Although these transient secondary structures in IDPs, which were recently named in 2012 as 
pre-structured motifs (PreSMos) (4), were first noticed in the late 1990 s $(2,3,17,18)$, they were not given the general name of PreSMos until structural details for a statistically significant number ( $\sim 4$ dozens) of MU type IDPs became available. Most well-known PreSMos are the amphipathic helix and two turns found in the 73-residue long transactivation domain (TAD) of tumor suppressor p53 (3). These PreSMos are the key determinants that enable binding of p53TAD to mdm2 (19), p62 (20), RPA (21) as well as the NCBD of CBP (22) and $\mathrm{BCl}-2$ (23). In other words, the PreSMos of p53 TAD are the "active sites" that are pre-populated transient secondary structures primed for target-binding. These PreSMos found in the p53 TAD, and other PreSMos observed in various IDPs/IDRs $(4,24-26)$ are likely to be at least part of the long-sought answer to the question of how IDPs/IDRs bind to various targets including proteins, nucleic acids, lipids and metals.

The glucocorticoid receptor (GR), a member of the nuclear receptor family, contains independent domains for DNA-binding and transactivation (27). Human GR contains two transactivation domains (TADs), one at its $\mathrm{N}$-terminus (tau1) and the other at its C-terminus (tau2) (28). The former consists of residues 77-262 (186-residues) and is responsible for the major transcriptional activity of hGR. We have previously shown that the minimal ("core") domain of tau1 containing residues 187-244 (called tau1c hereafter) retained $\sim 70 \%$ of the transactivation activity of the intact tau1 (29). hGR tau1c was one of the IDPs that was studied in the early days before the PreSMo concept was introduced. Since many transcription factors and TADs contain PreSMos, we wanted to learn whether tau1c also contains PreSMos. A previous NMR study on hGR tau1c indicated that it was largely unstructured in an aqueous solution, but it formed three helices under a helixinducing solvent, trifluoroethanol (TFE) (30). To determine whether the helices observed under TFE may be considered as PreSMos, we performed multi-dimensional NMR experiments on hGR tau1c in aqueous solvents using a ${ }^{15} \mathrm{~N} /{ }^{13} \mathrm{C}$-double labeled hGR tau1c.

\section{RESULTS}

Fig. 1 shows a fingerprint region of an ${ }^{15} \mathrm{~N}-{ }^{1} \mathrm{H}$ heteronuclear single quantum coherence (HSQC) spectrum of hGR tau1c with resonance assignment. Based on the narrow chemicalshift dispersion in both ${ }^{15} \mathrm{~N}$ and ${ }^{1} \mathrm{H}$ dimensions, we can confirm its overall unfolded/disordered nature in agreement with the bioinformatics prediction (Suppl. Fig. S1). Using the standard triple-resonance assignment procedure, we achieved a full NMR resonance assignment for the backbone ${ }^{15} \mathrm{~N}$ and the amide protons of the 64-residue hGR tau1c excluding the first two N-terminal residues, Met, and His, that originated from the $\mathrm{N}$-terminal glutathione-S-transferase fusion linker. For

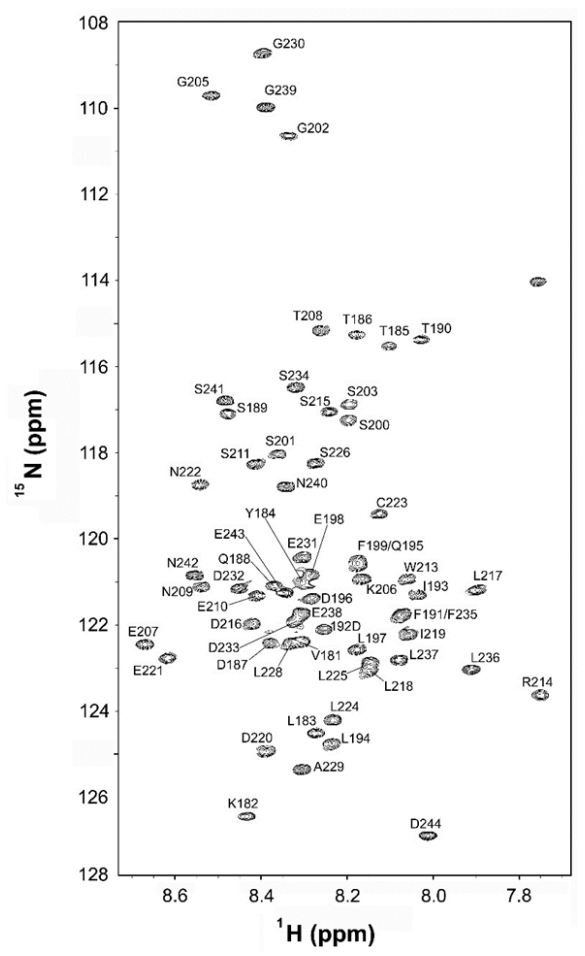

Fig. 1. A fingerprint region in an ${ }^{15} \mathrm{~N}-{ }^{1} \mathrm{H}$ HSQC spectrum of the $\mathrm{N}$-terminal region of hGR tau1c (residues 181-244) obtained at $10^{\circ} \mathrm{C}$ and $\mathrm{pH} 6.5$ on $90 \% \mathrm{H}_{2} \mathrm{O} / 10 \% \mathrm{D}_{2} \mathrm{O}$.

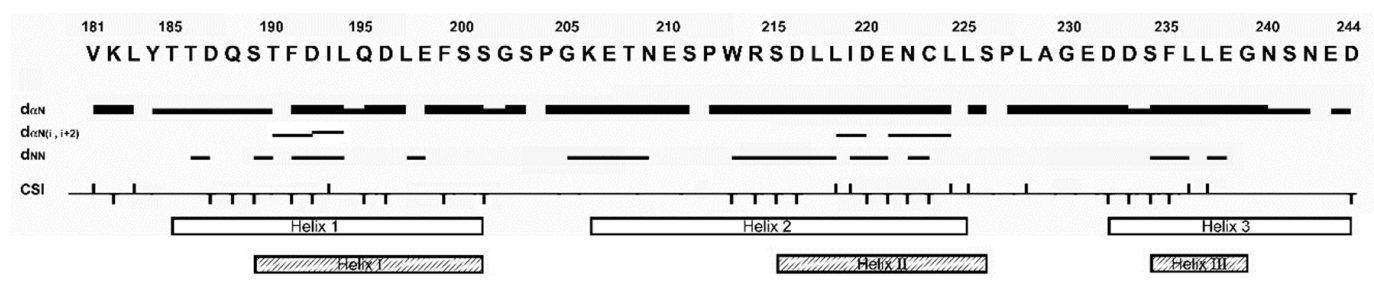

Fig. 2. The amino acid sequence of hGR tau1c. tau1c is shown along with the associated interproton NOEs and chemical shift indices (CSI). Continuous $\mathrm{d}_{\mathrm{NN}}$ interproton NOEs and CSIs are observed for three PreSMo regions of hGR tau1c, indicating their pre-structured (non-random) nature with transient helices. See the text for details. The three helix PreSMos identified in this study and those reported previously are shown as open and hatched boxes. 
3 prolines which do not have backbone amide $\mathrm{NH}$ protons, their aliphatic protons were fully assigned. The resonance assignment of hGR tau1c summarized in Table S1 was sufficient for structural characterization of the hGR tau1c, i.e., delineation of the PreSMo-forming residues.

Fig. 2 shows the amino acid sequence of hGR tau1c along with the associated interproton NOEs and chemical shift indices (CSI). Continuous dNN interproton NOEs from a NOESY spectrum (a mixing time of $150 \mathrm{~ms}$ ) and CSls are observed for three regions of the hGR tau1c, indicating the pre-structured (non-random) nature of hGR tau1c with several helix-forming residues. A quick examination of Fig. 2 reveals that the location of the three helices detected in the current study mostly overlaps with those previously detected under TFE. Fig. 3 shows several NMR parameters measured for hGR tau1c. Whether a PreSMo exists or not is usually determined by a combination of all available NMR parameters $(25,31)$. The left panel in Fig. 3 shows a summary of the chemical shift and deviations from random chemical shift values and SSP (secondary structure propensity) scores. The SSP scores were
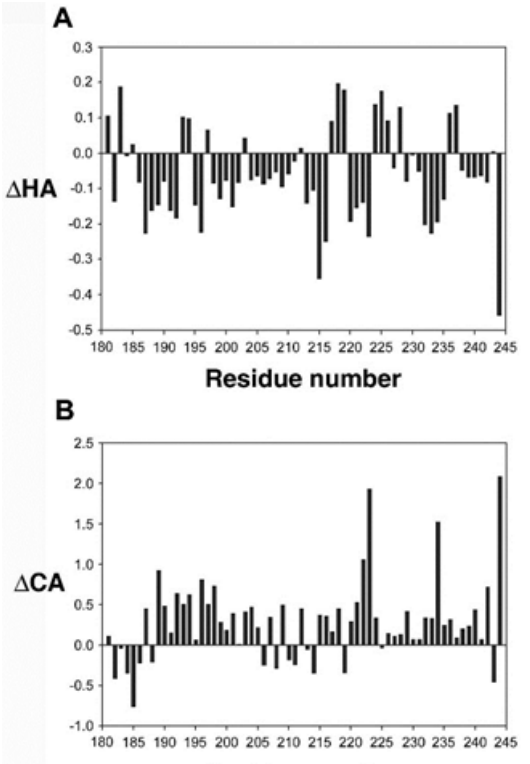

C

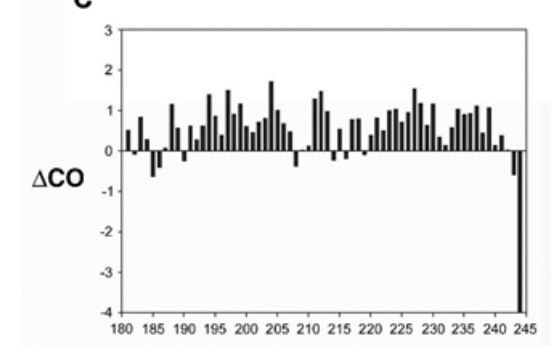

$$
\text { D }
$$

Residue number

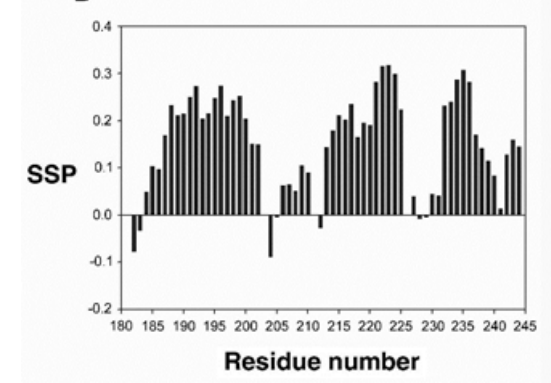

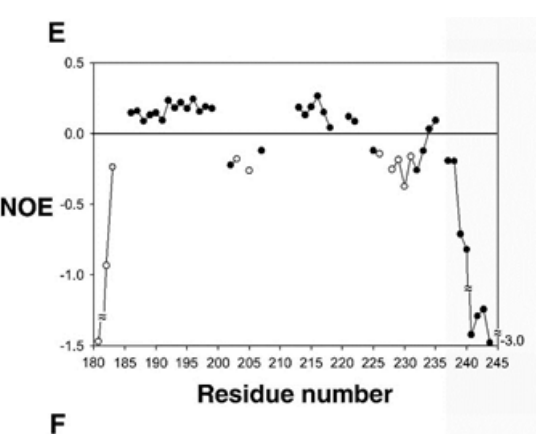

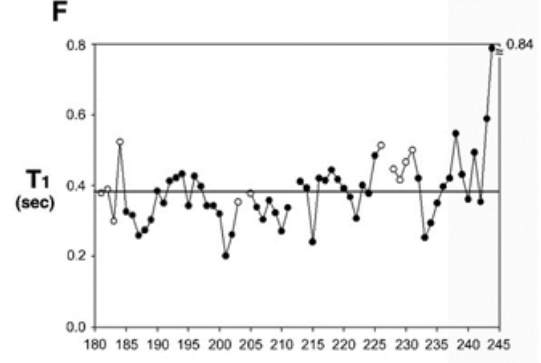

Residue number

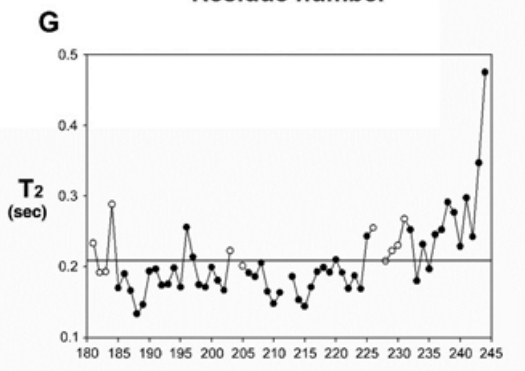

H

Residue number

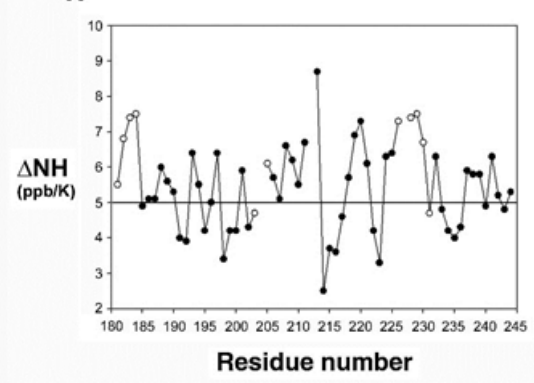

Fig. 3. Left panel: deviation of (A) ${ }^{1} \mathrm{H} \alpha$, (B) ${ }^{13} \mathrm{C} \alpha$, (C) carbonyl chemical shifts from random coil values and (d) the SSP (secondary structure propensity) scores of hGR tau1c (181-244). In (d), positive scores indicate helical propensity while negative values suggest formation of non-helical type PreSMos. Right panel: ${ }^{1} \mathrm{H}^{15} \mathrm{~N}$ heteronuclear NOEs $(\mathrm{E})$, backbone ${ }^{15} \mathrm{~N}$ relaxation times, $\mathrm{T}_{1}$ (F), $T_{2}(\mathrm{G})$, and temperature coefficients of the backbone amide hydrogens $(H)$. The horizontal lines in $(F)$ and $(G)$ indicate an average value. In $(\mathrm{H})$, temperature coefficients less than $5 \mathrm{ppb} / \mathrm{K}$ suggests formation of a helix. 
obtained by combining various chemical shifts $(\mathrm{H} \alpha, \mathrm{C} \alpha, \mathrm{C} \beta)$ (32) to provide the degree of pre-population of a PreSMo. The SSP values are often more conclusive about the location of a PreSMo than individual chemical shift deviations. Positive SSP scores over 4 residues or more indicate the formation of a helix, whereas negative values suggest non-helical ( $\beta$-type) secondary structures. Fig. 3d shows that hGR tau1c contains three helical PreSMos, the first (Helix 1) formed by residues 185-202, the second (Helix 2) by residues 206-225, and the third (Helix 3) by residues 232-244. All helices are $\sim 20-30 \%$ pre-populated. For most PreSMos positive (0.0-0.5), heteronuclear NOEs were observed. However, some reported heteronuclear NOEs for PreSMos were non-positive, being zero or slightly negative $(3,31)$.

\section{DISCUSSION}

By definition, PreSMos are transient or nascent secondary structures (average population of $\sim 30 \%$ ) detected by NMR in aqueous solution (4). With the discovery of PreSMos, we can classify IDPs/IDRs into two subclasses, a CU type and an MU type. Although several NMR parameters are used in combination to accurately delineate the residues that form a PreSMo, the SSP scores alone allow one to quickly judge if any IDP/IDR is a CU type or an MU type $(3,25,33,34)$. The SSP scores of hGR tau1c clearly indicate that it is an MU type, and the combination of all NMR parameters show that it forms three helical PreSMos: the first helix (Helix 1) formed by residues 185-202, the second (Helix 2) by residues 206-225, and the third (Helix 3 ) by residues 232-244, respectively. The three helices previously observed under TFE were Helix I (189-201), Helix II (215-226) and Helix III (234-239), respectively (30). Even though the three helices delineated in this study reasonably overlap with the three helices observed under TFE, notable differences exist at the N-termini of Helix 1 and Helix 2 and at the C-terminus of Helix 3.

For example, the first residue of Helix 1 is $185 \mathrm{~T}$ whereas the first one in Helix I is 189S. This difference can be ascribed to the fact that the starting residue of hGR tau1c is $181 \mathrm{~V}$ while that in the previous work was 188Q. Thus, it was not possible to observe the potential helix formation by residues 181-187 in the previous work. Another significant difference is noted at the $\mathrm{N}$-terminus of Helix 2. Helix 2 determined in the current study has a 9-residue longer $\mathrm{N}$-terminal portion than Helix II, and its N-terminus starts at residue $206 \mathrm{~K}$ instead of $215 \mathrm{~S}$. The previous work used only interproton NOEs since the SSP scores were introduced only in 2006. The usage of TFE must have generated helices in a higher population so that the interproton NOEs were stronger than those observed in the current study when we compare Fig. 2 of this report with Fig. 3 in the previous report (30). Previous data indicated that $213 \mathrm{~W}$ is important for transcriptional activity (35). Even though this residue was not a part of Helix II, a thorough reexamination of the data insinuates that $213 \mathrm{~W}$ may belong to
Helix II. It would be interesting to see if the inclusion of this bulky hydrophobic residue in a helical conformation would better explain the activity of hGR tau1c. Since the residue at 212 is a proline in Helix 2, it might introduce a kink just prior to $213 \mathrm{~W}$. Another difference is that the C-terminus of Helix 3 is longer by 5 residues than Helix III. It also is very interesting to learn that two prolines, 204P and 226P, flank the C-termini of the two helices, Helix 1 and Helix 2. The helix-flanking prolines of helix PreSMos that might act as a subtle activity switch were described earlier (36).

Tau1c of hGR is one of the IDRs that was studied very early in IDP research when controversy was keen regarding the question of whether transactivation domains should contain some sort of specificity determinants that mediate target binding (3). Although hGR tau1c was shown to form three helices that might be important for transcriptional activity (30), this pioneering data was not considered when the PreSMo concept was formulated (4) since the observation of helices was made in TFE that may artificially induce helix formation. The current study was initiated to re-investigate this IDR in aqueous solvents in order to determine if it forms PreSMos and if it should be classified as an MU type. We confirmed that hGR tau1c is an MU type IDR. Our work demonstrated that the PreSMo concept is further expandable to other IDPs/IDRs, including the IDPs studied before the introduction of the PreSMos concept. Recent mutation studies on p53 TAD and CNBR $(37,38)$ have elegantley shown that the degree of pre-population of a helix PreSMo is critical for target binding, suggesting the degree of PreSMo pre-population is a variable inherent in the MU type IDPs/IDRs that is quite subtly tuned for target binding. It would be interesting to see if one can also establish such a correlation between the degree of helix pre-population and the activity of hGR tau1c.

\section{MATERIALS AND METHODS}

Detailed experimental and computational procedures are described in the Supplementary Information.

\section{ACKNOWLEDGEMENTS}

This work was supported by a Korea-Hungary and Pan EU collaborative project from the National Research Council of Science and Technology (NST) (NTC2251422) of Korea (to KH \& AW) as well as grants from the Swedish Research Council and Swedish Cancer Society (to AW).

\section{CONFLICTS OF INTEREST}

The authors have no conflicting interests.

\section{REFERENCES}

1. The RCSB Protein Data Bank (https://www.rcsb.org)

2. Daughdrill GW, Chadsey MS, Karlinsey JE, Hughes KT, 
Dahlquist FW (1997) The C-terminal half of the anti-sigma factor, FlgM, becomes structured when bound to its target, $\sigma^{28}$. Nat Struct Mol Biol 4, 285-291.

3. Lee $H$, Mok KH, Muhandiram R et al (2000) Local structural elements in the mostly unstructured transcriptional activation domain of human p53. J Biol Chem 275, 29426-29432

4. Lee S-H, Kim D-H, Han JJ et al (2012) Understanding pre-structured motifs (PreSMos) in intrinsically unfolded proteins. Curr Protein Pept Sci 13, 34-54

5. Dunker AK, Babu MM, Barbar E et al (2013) What's in a name? Why these proteins are intrinsically disordered. Intrinsically Disordered Proteins 1, e24157

6. Uversky VN, Oldfield CJ, Dunker AK (2008) Intrinsically disordered proteins in human diseases: Introducing the D2 concept. Annu Rev Biophys 37, 215-246

7. Tompa P, Han KH, Bokor M et al (2016) Wide-line NMR and DSC studies on intrinsically disordered p53 transactivation domain and its helically pre-structured segment. BMB Rep 9, 497-501

8. Eliezer D, Kutluay E, Bussell R Jr, Browne G (2001) Conformational properties of $\alpha$-synuclein in its free and lipid-associated states. J Mol Biol 307, 1061-1073

9. Mukrasch MD, Bibow S, Korukottu J et al (2009) Structural polymorphism of 441-residue tau at single residue resolution. PLoS Biol 7, e1000034

10. Chi SW, Kim DH, Lee SH, Chang I, Han KH (2007) Pre-structured motifs in the natively unstructured preS1 surface antigen of hepatitis B virus. Protein Sci 16, 2108-2117

11. Kim DH, Lee SH, Nam KH, Chi SW, Chang I, Han KH (2009) Multiple hTAFII31-binding motifs in the intrinsically unfolded transcriptional activation domain of VP16. BMB Rep 42, 411-417

12. Lee SH, Cha EJ, Lim JE et al (2012) Structural characterization of an intrinsically unfolded mini-HBX protein from hepatitis B virus. Mol Cells 34, 165-169

13. Xue B, Blocquel D, Habchi J et al (2014) Structural disorder in viral proteins. Chem Rev 114, 6880-6911

14. Lee C, Kim DH, Lee SH, Su J and Han KH (2016) Structural investigation on the intrinsically disordered $\mathrm{N}$-terminal region of HPV16 E7 protein. BMB Rep 49, 431-436

15. Radhakrishnan I, Pérez-Alvarado GC, Parker D, Dyson HJ, Montminy MR, Wright PE (1997) Solution structure of the KIX domain of CBP bound to the transactivation domain of CREB: A model for activator:coactivator interactions. Cell 91, 741-752

16. Fletcher CM and Wagner G (1998) The interaction of elF4E with $4 \mathrm{E}-\mathrm{BP} 1$ is an induced fit to a completely disordered protein. Protein Sci 7, 1639-1642

17. Ramelot TA, Gentile LN, Nicholson LK (2000) Transient structure of the amyloid precursor protein cytoplasmic tail indicates preordering of structure for binding to cytosolic factors. Biochemistry 39, 2714-2725

18. Sayers EW, Gerstner RB, Draper DE, Torchia DA (2000) Structural preordering in the $\mathrm{N}$-terminal region of ribosomal protein $\mathrm{S} 4$ revealed by heteronuclear NMR spectroscopy. Biochemistry 39, 13602-13613

19. Chi SW, Lee SH, Kim DH (2005) Structural details on mdm2-p53 interaction. J Biol Chem 280, 38795-38802
20. Di Lello P, Jenkins LMM, Jones TN et al (2006) Structure of the Tfb1/p53 complex: Insights into the interaction between the p62/Tfb1 subunit of TFIIH and the activation domain of p53. Mol Cell 22, 731-740

21. Bochkareva E, Kaustov L, Ayed A et al (2005) Singlestranded DNA mimicry in the p53 transactivation domain interaction with replication protein A. Proc Natl Acad Sci U S A 102, 15412-15417

22. Lee CW, Martinez-Yamout MA, Dyson HJ, Wright PE (2010) Structure of the p53 transactivation domain in complex with the nuclear receptor coactivator binding domain of CREB binding protein. Biochemistry 49, 9964-9971

23. Ha JH, Shin JS, Yoon MK (2013) Dual-site interactions of p53 protein transactivation domain with anti-apoptotic $\mathrm{Bcl}-2$ family proteins reveal a highly convergent mechanism of divergent p53 pathways. J Biol Chem 288, 7387-7398

24. Andresen C, Helander S, Lemak A et al (2012) Transient structure and dynamics in the disordered c-Myc transactivation domain affect Bin1 binding. Nucleic Acids Res 40, 6353-6366

25. Kim DH, Lee C, Cho YJ (2015) A pre-structured helix in the intrinsically disordered 4EBP1. Mol BioSyst 11, 366-369

26. Berlow RB, Dyson HJ1, Wright PE (2017) Hypersensitive termination of the hypoxic response by a disordered protein switch. Nature 543, 447-451

27. Evans RM (1988) The steroid and thyroid hormone receptor superfamily. Science 240, 889-895

28. Hollenberg SM and Evans RM (1988) Multiple and cooperative trans-activation domains of the human glucocorticoid receptor. Cell 55, 899-906

29. Dahlman-Wright K, Almlöf T, McEwan IJ, Gustafsson JA, Wright AP (1994) Delineation of a small region within the major transactivation domain of the human glucocorticoid receptor that mediates transactivation of gene expression. Proc Natl Acad Sci U S A 91, 1619-1623

30. Dahlman-Wright K, Baumann H, McEwan IJ et al (1995) Structural characterization of a minimal functional transactivation domain from the human glucocorticoid receptor. Proc Natl Acad Sci U S A 92, 1699-1703

31. Kim DH, Lee C, Lee SH et al (2017) The Mechanism of p53 Rescue by SUSP4. Angew Chem Int Ed Engl 56, 1278-1282

32. Marsh JA, Singh VK, Jia Z, Forman-Kay JD (2006) Sensitivity of secondary structure propensities to sequence differences between $\alpha$ - and $\gamma$-synuclein: Implications for fibrillation. Protein Sci 15, 2795-2804

33. Baker JM, Hudson RP, Kanelis V et al (2007) CFTR regulatory region interacts with NBD1 predominantly via multiple transient helices. Nat Struct Mol Biol 14, 738-745

34. Zhang X, Perugini MA, Yao S et al (2008) Solution conformation, backbone dynamics and lipid interactions of the intrinsically unstructured malaria surface protein MSP2. J Mol Biol 379, 105-121

35. Almlöf T, Gustafsson JA, Wright AP (1997) Role of hydrophobic amino acid clusters in the transactivation activity of the human glucocorticoid receptor. Mol Cell 
Biol 17, 934-945

36. Lee C, Kalmar L, Xue B et al (2014) Contribution of proline to the pre-structuring tendency of transient helical secondary structure elements in intrinsically disordered proteins. Biochim Biophys Acta 1840, 993-1003

37. Borcherds W, Theillet FX, Katzer A et al (2014) Disorder and residual helicity alter p53-Mdm2 binding affinity and signaling in cells. Nat Chem Biol 10, 1000-1002

38. lešmantavičius $V$, Dogan J, Jemth $P$, Teilum $K$, Kjaergaard M (2014) Helical propensity in an intrinsically disordered protein accelerates ligand binding. Angew Chem Int Ed Engl 53, 1548-1551 